dementias and the subcommittee has recommended that the MRC should give priority to support for suitable projects.

Meantime, what can we do for these patients and their unfortunate families? Doctors, nurses, and social workers need to be aware of the importance of early recognition so that caring relatives (and neighbours) should receive the best counselling and support. There are five demented patients in the community for every one in hospital, ${ }^{1}$ and emphasis should be given to community care by providing adequate health visitor, home nursing, and home help services together with good day care facilities. These community services must be backed up by adequate institutional services, and prompt admission should be available at the time of need, whether it be due to deterioration in the patient's condition or a call for a respite for caring relatives.

Strict attention to general health is important for patients with dementia: any intercurrent illness leads to rapid deterioration and dependency, often with increased confusion and behaviour disorder. At all costs families must be protected from reaching a state of exhaustion and despair, because rejection of an old person by his or her family is almost always irreversible. ${ }^{12}$ Monitoring the state of the family is often best undertaken by the health visitor, though where a social worker is already closely concerned the task may be left to her. At all costs a multiplicity of visitors (often with a minimum of effective action) should be avoided-this only increases frustration and bewilderment.

Many drugs are marketed with alleged beneficial effects on mental function in demented patients, but psychiatrists and geriatricians remain unconvinced of their value. Minor and major tranquillisers, however, are extremely useful for controlling restlessness, insomnia, and aggressive behaviour. Each practitioner should choose his own small range of drugs and get to know them well by using them in carefully titrated dosage with frequent review and a willingness to withdraw or reduce dosage as the patient improves.

At present responsibility for institutional care is divided among the local authority, the psychiatric services, and the geriatric services. Inevitably, too, some patients find their way into acute hospital wards. This confusion is inimical to the efficient use of resources, and services need to be co-ordinated properly to ensure that patients are in the right institution at the right time. The use of special local authority hostels for the elderly with dementia has not been widely accepted; in Scotland local authorities have avoided making any provision at all. The Royal College of Psychiatrists (Scottish Division) recently produced a short report on the psychiatrist's contribution to the care of the elderly, firmly recommending that the NHS should provide this institutional care for elderly demented patients and that local authorities should be freed from this responsibility. Firm decisions on these matters are long overdue. Meanwhile, scarce resources are being squandered by failure to take the necessary steps towards rationalisation. Failure to tackle the increasing problem of dementia will not merely jeopardise community services: it will threaten the working of all NHS institutions, including the acute hospital sector.

\footnotetext{
1 Kay, D W K, Beamish, P, and Roth, M, British Fournal of Psychiatry, 1964, 110, 146.

2 Kay, D W K, et al, Comprehensive Psychiatry, 1970, 11, 26.

3 Medical Research Council, Senile and Presenile Dementias. London, Medical Research Council, 1977.

4 Blessed, G, Tomlinson, B E, and Roth, M, British fournal of Psychiatry, 1968, 114, 797.

5 Larsson, T, Sjögren, T, and Jacobson, G, Acta Psychiatrica Scandinavica, 1963, suppl 167.

- Hachinski, V C, Lassen, N A, and Marshall, J, Lancet, 1974, 2, 207.
}

7 Perry, T L, Hansen, S, and Kloster, M, New England fournal of Medicine, 1973, 288, 337.

${ }^{8}$ Davies, P, and Maloney, A F J, Lancet, 1976, 2, 1403.

Gajdusek, D C, and Zigas, V, New England fournal of Medicine, 1957, 257, 974.

10 Gibbs, C J, et al, Science, 1968, 161, 388.

11 Duffy, P, et al, New England fournal of Medicine, 1974, 290, 692.

12 MacMillan, D, Lancet, 1960, 2, 1439.

\section{Legionnaires' disease}

It is years since a new disease has been recognised which proves to have a bacterial cause; we have long learned to expect that an infection of unknown aetiology will be due to a virus or rickettsia. Yet such is legionnaires' disease, first recognised-although it is now known to have occurred before -when it took epidemic form at a convention in Philadelphia in 1976. Hitherto many subsequent studies, not only of further American cases but also others in British visitors to Benidorm and of some in Nottingham, have been briefly reported in epidemiological bulletins-findings we reviewed recently ${ }^{1}$ but a full account of last year's outbreak and of the work which disclosed its cause has now appeared in two papers from the Center for Disease Control, Atlanta. ${ }^{2} 3$

The Pennsylvania State American Legion Convention at Philadelphia on 21-24 July was attended by 3683 delegates and others, among whom 183 were attacked, with 29 deaths. Incidence increased with age, from $3.7 \%$ in the under- 40 s to $12.3 \%$ in the over-70 group. The incubation period was about seven days, although sometimes longer. At the onset a day of malaise and muscle pain was followed by rapidly rising fever to a high maximum, with rigors, cough (at first dry), chest pain, and dyspnoea. Cyanosis is not mentioned, but most patients were treated with oxygen and some required mechanical ventilation. There was proteinuria, often some impairment of renal function, a moderate leucocytosis, and a raised sedimentation rate. Radiography showed patchy consolidation proceeding to confluence, which in nearly half the cases was unilateral. After two or three days' progression resolution was by lysis. Of seven antibiotics cephalothin appeared to be the least and tetracycline and erythromycin the most effective; the efficacy of erythromycin has since been confirmed experimentally.

The source and mode of spread of the infection were closely investigated, with mainly negative and in some ways puzzling results. Although infection was believed to be air-borne, person-to-person spread was not observed, and in the principally affected hotel (" $A$ ") housing the delegates further cases did not occur after the convention was over. The only employee of 400 in the hotel possibly but not certainly affectedand this on a vital date (24 July) - was an air-conditioner repair man, but in $29 \%$ of the staff serological evidence of past infection was obtained later, and it is believed that the infection may be mild or inapparent. Minute inquiries about the detailed movements of "conventioneers" showed that those affected had spent longer in the lobby of hotel A. Here the story becomes confused, because some of those attending three other functions were affected (a eucharistic congress and conventions of candlemakers and magicians), and there was a simultaneous outbreak of "Broad Street pneumonia," which appears to have been the same disease. For non-residents of hotel A the authors claim to have established a connection between attack rate and the time spent on the pavement outside the hotel watching a parade; a distinction is even drawn 
between those who had been on opposite sides of the street. This suggestion of air-borne infection in the open air seems difficult to credit. The operation of some other factor may be suspected, unidentifiable long after the event.

If this aspect of the disease remains obscure we have no doubt about its cause. Comprehensive laboratory studies disclosed no evidence of infection by any agent hitherto known to cause pneumonia. The positive results were obtained with specimens of lung from fatal cases. Films and cultures of these showed no significant bacteria, but of six guinea-pigs inoculated intraperitoneally with lung suspension four developed a febrile infection fatal in three to six days; one of these lungs was from a case of Broad Street pneumonia. Examination of peritoneal exudate, spleen, liver, and lung stained by silver impregnation showed numerous bacilli; an illustration shows these as varying in length and thickness with tapering ends; long threads were also present. This organism grew freely in the yolk sac of the chick embryo, but was non-pathogenic for mice. It was Gram-negative (by which is apparently meant that by Gram stain it was invisible) and non-acid-fast. It has since been cultivated on a common agar medium with supplements, but its colonial characteristics are not described.

Yolk-sac suspensions. were used for indirect fluorescence antibody tests. In these, dried films are treated first with dilutions of serum from a suspected case, and then with rabbit anti-human globulin serum conjugated with fluorescein; bacilli with which antibody has combined are then brightly stained. This test not only confirmed the presence of rising and high titre antibody in convalescent sera and its absence in control sera, but, thanks to the preservation of serum specimens in duplicate (acute and convalescent phase) from earlier epidemics and individuals, furnished proof that the disease is far from new. An epidemic in a mental hospital in the District of Columbia in 1965, with 89 cases and 12 deaths, was clearly of the same nature, and another in a county health department in Pontiac, Michigan, in 1968, with no deaths, was identified as a mild form of the disease. Oddly, these two as well as the Philadelphia outbreak occurred in the summer. Nine recently seen patients with sporadic pneumonia in six different states also showed seroconversion. This test has, of course, also been responsible for verifying the nature of recent cases in Spain and Britain.

Here, then, we have a new form of pneumonia, the source of the infection being unknown, and with apparently no characteristic clinical features. It may be suspected if no bacterium or virus can be found, but how can it be diagnosed short of sending serum to Atlanta? Doubtless cultures will come to Britain and enable us to perform the fluorescent antibody test here. But we should like to know whether a more direct method may be feasible. All studies of the bacillus seem to have been carried out so far with lungs from fatal cases. When further opportunities occur attention might well be directed to the sputum; presumably the silver impregnation method of staining which showed the organism in guinea-pigs has yet to be applied to this material. Even cultivation of the organism may be possible, for it has been achieved on a conventional medium. Apart from the question of diagnostic convenience, bacteriologists would dearly like to know much more about the bacillus itself; on present information it cannot be assigned to a genus or even classified more broadly. It also remains to be discovered where it exists between episodes of active infection.

\footnotetext{
1 British Medical fournal, 1977, 2, 1425.

${ }^{2}$ Fraser, D W, et al, New England fournal of Medicine, 1977, 297, 1189.

${ }^{3}$ McDade, J E, et al, New England Fournal of Medicine, 1977, 297, 1197.
}

\section{Coeliac disease in Galway}

Our definition of coeliac disease still depends on the response to treatment. A coeliac is someone whose small-intestinal mucosa is genetically predisposed to damage from exposure to gluten. The abnormality persists throughout life, unlike the usually less severe lesion of transient gluten intolerance found in some children. ${ }^{2}$ The intestinal damage should be shown to improve substantially after gluten withdrawal. When the diagnosis is still in doubt, a third intestinal biopsy may be needed to show that the damage recurs after a gluten challenge.

Participants at the Third International Coeliac Symposium held recently in the beautiful setting of Galway seemed agreed on these points. Nevertheless, the genetic pattern, the toxic fraction of gluten, the diagnostic mucosal lesion, and the criteria for response to gluten withdrawal and to gluten challenge all remain undefined and open to debate. Such discussion is the meat and drink of symposia and there was plenty of all three ingredients.

The histocompatibility antigens HLA B8 and especially HLA DW3 are associated with coeliac disease. ${ }^{34}$ An even closer association seems to be found with a specific B-lymphocyte-associated antigen. ${ }^{5}$ As Strober and his colleagues from Bethesda suggested, there may be two genes coding for proteins which make up a single structure on the surface of B cells. Such a structure might be a receptor for gliadin, the toxic fraction of gluten.

The meeting was reminded by the workers from Leiden, where the important Second International Symposium was held in 1974, that gliadin itself is a heterogeneous protein currently defined by its electrophoretic mobility. It takes a great deal of time to obtain electrophoretically pure alphagliadin (which probably contains the toxic subfraction of gliadin) in sufficient quantities to use in clinical tests. Different centres use their own schedules for challenging the mucosa of the treated patient with coeliac disease. Most still use gluten, but the amount, presentation, and duration of the gluten challenge all vary. The symposium set up a small working group to try to agree a standard technique. One aspect of gluten challenge that provoked interest in physicians caring for adults was the different response of the treated coeliac child's mucosa to gluten. If the child remains symptom free on return to a normal diet re-exposure to gluten may be prolonged for two years or more before it induces significant mucosal damage. In contrast, changes can be induced in adult coeliacs given 20-30 g gluten or a few days' normal diet (containing about $12 \mathrm{~g}$ gluten daily). Some adult patients can hardly tolerate even this amount of gluten without gastrointestinal symptoms.

High hopes were raised by some elegant work on a variant of Chinese Spring wheat which lacks alpha-gliadins and hence could potentially be eaten by these patients. Unfortunately, predictions were not supported by evidence from intestinal biopsy studies, which alone could show whether patients can eat this wheat with impunity. The results of the home team's study of "detoxified" gliadin with a carbohydrate moiety removed also ran into difficulties at the conference, for many thought that the small biopsy specimens shown as evidence of lack of damage after challenge with this substance seemed to contain appreciable numbers of abnormal epithelial surface cells. By the end of the ensuing discussion the case for detoxification depended largely on the findings in one patient, and the verdict remained "not proven." 